\title{
Evolution and nucleosynthesis of extremely metal-poor and metal-free low- and intermediate-mass stars
}

\section{II. s-process nucleosynthesis during the core He flash}

\author{
S. W. Campbell ${ }^{1,2,3, \star}$, M. Lugaro ${ }^{3, \star}$, and A. I. Karakas ${ }^{4,3}$ \\ 1 Departament de Física i Enginyeria Nuclear, Universitat Politècnica de Catalunya, EUETIB, Carrer Comte d'Urgell 187, \\ 08036 Barcelona, Spain \\ e-mail: simon.w. campbell@upc . edu \\ 2 Institut de Ciències de l'Espai (ICE-CSIC), Campus UAB, Fac. Ciències, Torre C5 parell 2, 08193 Bellaterra, Spain \\ 3 Centre for Stellar and Planetary Astrophysics, Monash University, Clayton, VIC 3800, Australia \\ e-mail: maria.lugaro@monash.edu \\ 4 Research School of Astronomy and Astrophysics, Australian National University, Canberra, Australia \\ e-mail: akarakas@mso.anu.edu.au
}

Received 19 July 2010 / Accepted 6 October 2010

ABSTRACT

\begin{abstract}
Context. Models of primordial and hyper-metal-poor stars that have masses similar to the Sun are known to experience an ingestion of protons into the hot core during the core helium flash phase at the end of their red giant branch evolution. This produces a concurrent secondary flash powered by hydrogen burning that gives rise to further nucleosynthesis in the core.

Aims. We aim to model the nucleosynthesis occurring during the proton ingestion event to ascertain if any significant neutron-capture nucleosynthesis occurs.

Methods. We perform post-process nucleosynthesis calculations on a one-dimensional stellar evolution calculation of a star with mass $1 M_{\odot}$ and a metallicity of $[\mathrm{Fe} / \mathrm{H}]=-6.5$ that suffers a proton ingestion episode. Our network includes 320 nuclear species and 2366 reactions and treats mixing and burning simultaneously.

Results. We find that the mixing and burning of protons into the hot convective core leads to the production of ${ }^{13} \mathrm{C}$, which then burns via the ${ }^{13} \mathrm{C}(\alpha, n){ }^{16} \mathrm{O}$ reaction, releasing a large number of free neutrons. During the first two years of neutron production the neutron poison ${ }^{14} \mathrm{~N}$ abundance is low, allowing the prodigious production of heavy elements such as strontium, barium, and lead via slow neutron captures (the $s$ process). These nucleosynthetic products are later carried to the stellar surface and ejected via stellar winds. We compare our results with observations of the hyper-metal-poor halo star HE 1327-2326, which shows a strong Sr overabundance. Conclusions. Our model provides the possibility of self-consistently explaining the Sr overabundance in HE 1327-2326 together with its $\mathrm{C}, \mathrm{N}$, and $\mathrm{O}$ overabundances (all within a factor of $\sim 4$ ) if the material were heavily diluted, for example, via mass transfer in a wide binary system. The model produces at least 18 times too much Ba than observed, but this may be within the large modelling uncertainties. In this scenario, binary systems of low mass must have formed in the early Universe. If this is true, it puts constraints on the primordial initial mass function.
\end{abstract}

Key words. nuclear reactions, nucleosynthesis, abundances - stars: evolution - stars: individual: HE 1327-2326 - stars: interiors stars: Population II - stars: Population III

\section{Introduction}

Stars with a similar mass as the Sun eventually expand and become red giants, and at the end of their red giant phase they undergo a thermonuclear runaway in the core known as the core helium flash. The ignition of helium in this flash event occurs at the hottest place in the core, which is located off centre because of cooling via neutrino energy losses deep in the stellar interior. The amount of energy released by the He flash is so large that it cannot be carried radiatively, and thus convective motions are triggered, forming the so-called He convective zone (HeCZ). A typical $\mathrm{HeCZ}$ extends from the location of the He-burning region to close to the base of the H-burning shell. The convection does not normally break through the H-burning shell because the active H-burning shell provides an entropy barrier against mixing (Iben Jr. 1976).

* The first two authors have contributed equally to this paper.
However, in solar-mass red giant stars of primordial $(Z=0)$ or hyper-metal-poor $([\mathrm{Fe} / \mathrm{H}] \lesssim-5.0)$ composition the $\mathrm{H}-\mathrm{He}$ interface is not as impenetrable as in more metal-rich red giant stars. The reason for this is twofold. First, the core helium flash starts much farther away from the centre in these lowmetallicity models (at $\sim 0.27 M_{\odot}$ in the model we present here, Fig. 1) than at solar metallicity and is thus closer to the $\mathrm{H}$ shell. Second, the entropy barrier at the $\mathrm{H}$ shell is much weaker in stars of very low metallicity because the H-burning shell almost switches off at this stage of evolution (Fujimoto et al. 1990). Thus, in primordial solar-mass red giants the He convective region can penetrate into the overlying $\mathrm{H}$-rich region and protons can be carried down into the hot He-burning zone. If enough protons are ingested, a secondary (H-burning) "flash" will occur within the He convective region while the He-burning flash is still ongoing. This can result in the He-convective region splitting into two convective regions at the location where the $\mathrm{H}$ burning releases most of its energy. This sequence of events 
is well established in 1D stellar models and is referred to variously as "dual core flash" (DCF), "proton ingestion episode" or "helium-flash-induced mixing" (Fujimoto et al. 1990; Hollowell et al. 1990; Schlattl et al. 2001; Picardi et al. 2004; Campbell \& Lattanzio 2008; Suda \& Fujimoto 2010). Importantly, the material processed by the DCF is later carried to the stellar surface by a (single) dredge-up event. Thus the nucleosynthetic products of the DCF are available to be shed into the surrounding interstellar medium by stellar winds or transferred to a binary companion. The main chemical products are $\mathrm{C}$ and $\mathrm{N}$, and this qualitatively agrees with the observations of C-rich extremely metal-poor halo stars in the Galactic Halo (Schlattl et al. 2001; Picardi et al. 2004).

Here we explore in detail the consequences of a proton ingestion episode during the core He flash on the nucleosynthesis and surface abundances of a star of $1 M_{\odot}$ and $[\mathrm{Fe} / \mathrm{H}]=-6.5$.

\section{Method}

We have used one of the stellar structure models computed in Campbell \& Lattanzio (2008) (Paper I). This model was calculated using the Schwarzschild criterion to define the border of convective regions and a diffusive mixing treatment of the movement of material inside convective regions similar to that of Meynet et al. (2004). The stellar structure code follows six chemical species: ${ }^{1} \mathrm{H},{ }^{3} \mathrm{He},{ }^{4} \mathrm{He},{ }^{12} \mathrm{C},{ }^{14} \mathrm{~N}$, and ${ }^{16} \mathrm{O}$. The rest of the isotopes involved in the $\mathrm{CNO}$ cycles are included by assuming that they are always present in their equilibrium abundances.

We calculate the detailed nucleosynthesis with a postprocessing code that activates nuclear reactions in the star on the basis of the information on the temperature, density, and convective velocities provided by the stellar structure model (see Paper I and references therein for details). The equations that describe the changes in the abundances are solved using an implicit method so that a large matrix of $n^{2}$ is solved, where $n$ is the number of species in the network. Nuclear burning and convective mixing are both included in the equations of the abundance changes and thus solved simultaneously. Our present calculation was performed using a nuclear network of $n=320$ species, from protons and neutrons up to lead and bismuth, and 2336 reactions with rates from the JINA reaclib database (Cyburt et al. 2010). The initial composition for the elements up to $\mathrm{Zn}$ was taken as a mix of standard Big Bang material and supernova calculations as described in Paper I. The abundances of the elements heavier than $\mathrm{Zn}$ were taken equal to zero, however we note that this choice is mostly irrelevant for our results because the neutron-capture nucleosynthesis overwrites the memory of the initial abundances.

Owing to our computationally expensive scheme we only included some of the $s$-process branching points that may be activated under conditions of a high neutron flux. This shortcoming should not have a strong impact on the overall heavy-element distribution reported here because the overall neutron flux is only very marginally affected by the details of the $s$-process path.

Due to computational problems we have not yet proceeded much past the end of the neutron superburst. However, this did not prevent us from accurately estimating the surface composition of the neutron-capture elements for the model (Sect. 3.2). This is because there are no further mixing episodes following the dredge-up event that mixes the products of the core He flash into the stellar envelope.

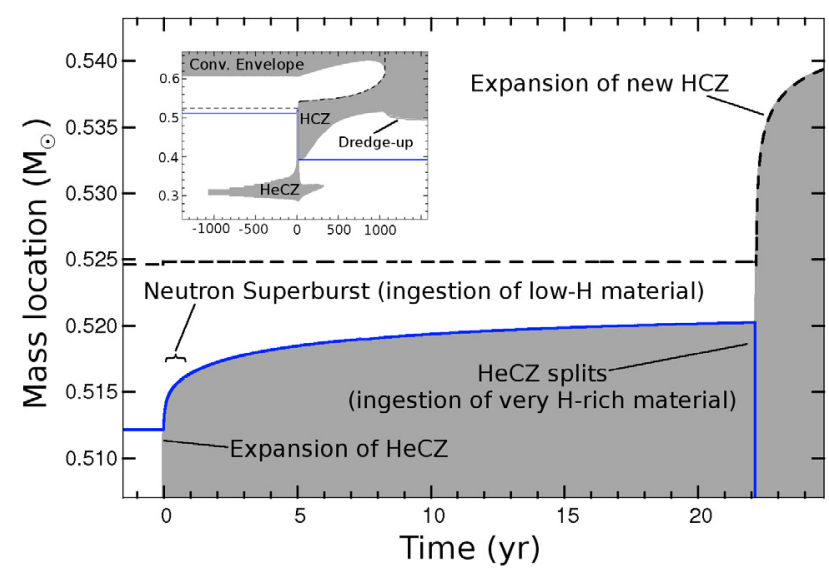

Fig. 1. Internal evolution of our solar-mass model star with a metallicity of $[\mathrm{Fe} / \mathrm{H}]=-6.5$ during the core He flash. Proton ingestion starting at $t=0(6.3 \mathrm{Gyr}$ into the stellar evolution) owing to the expansion of the $\mathrm{HeCZ}$ leads to the production of ${ }^{13} \mathrm{C}$ and the consequent neutron superburst via ${ }^{13} \mathrm{C}(\alpha, n){ }^{16} \mathrm{O}$. Convective regions are grey, $\mathrm{HeCZ}: \mathrm{He}$ convective zone, HCZ: H convective zone. The blue solid line and the black dashed line represent the location in mass where the abundance of $\mathrm{H}$ is 0.0001 and 0.73 , respectively. The insert is a zoomed-out view to help localize the region of interest within the star as a whole. It can be seen that some of the processed material starts to be dredged up by the encroaching convective envelope at around $t=1000 \mathrm{yr}$.

\section{Results}

\subsection{The "neutron superburst"}

We find that our detailed DCF model experiences a phase of very high neutron flux in the He-rich convective region, which we call a neutron "superburst". The neutron flux for the superburst is primarily supplied by ${ }^{13} \mathrm{C}(\alpha, n){ }^{16} \mathrm{O}$ reactions occurring at the base of the HeCZ. The ${ }^{13} \mathrm{C}$ for this reaction is produced as a consequence of the proton ingestion - it is a product of partial $\mathrm{CN}$ cycling via ${ }^{12} \mathrm{C}(p, \gamma)^{13} \mathrm{~N}\left(\beta^{+}\right)^{13} \mathrm{C}$, where the ${ }^{12} \mathrm{C}$ is supplied by the on-going triple- $\alpha$ reactions of the He-flash (Campbell 2007; Lugaro et al. 2009). We note that Fujimoto et al. (1990) speculated that neutrons and light $s$-process elements may be produced during this event.

The superburst occurs during the earliest phases of the ingestion (Fig. 1). This is because the $\mathrm{H}$ profile is very extended (Fig. 2) owing to the nature of the previous H-shell burning. In a star with a negligible amount of CNO catalysts the p-p chain reactions dominate over the $\mathrm{CNO}$ cycles in terms of the energy production. Because the $\mathrm{p}-\mathrm{p}$ chains have a low temperature dependence, the H-burning shell is active over an extended region, leading to a broadened $\mathrm{H}$-abundance profile. This long $\mathrm{H}$ "tail" leads to variations in the amount of protons ingested into the expanding $\mathrm{HeCZ}$. In the early ingestion phases, the mixing of small amounts of protons mostly lead to the production of ${ }^{13} \mathrm{C}$ nuclei and the consequent neutron superburst $(t=0$ to $\sim 1.2 \mathrm{yr}$ in Fig. 2). After about $1.7 \mathrm{yr}$, the $\mathrm{HeCZ}$ has expanded through the entire $\mathrm{H}$ abundance profile up to the base of the unburnt envelope. At this point the abundance of protons in the $\mathrm{HeCZ}$ becomes so high that the abundance of the neutron poison ${ }^{14} \mathrm{~N}$ exceeds that of ${ }^{13} \mathrm{C}$ owing to complete $\mathrm{CN}$-cycling. Under these conditions the ${ }^{14} \mathrm{~N}(n, p){ }^{14} \mathrm{C}$ reactions dominate and the neutron superburst is quenched.

The neutron density during the superburst peaks at $\sim 10^{15} \mathrm{n} \mathrm{cm}^{-3}$, remains above $10^{14} \mathrm{n} \mathrm{cm}^{-3}$ for about 0.2 years (Fig. 3), and the time-integrated neutron flux is $287 \mathrm{mbarn}^{-1}$. At 


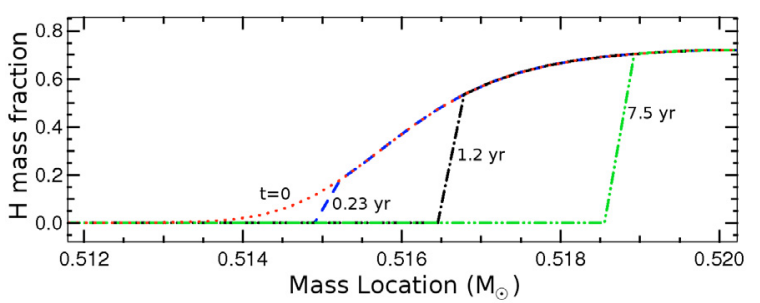

Fig. 2. The H profile around the location of the proton ingestion at various times during the neutron superburst $(t=0$ corresponds to the same time in Fig. 1). The peak neutron flux occurs between $t \sim 0$ and $t \sim 1.2 \mathrm{yr}$.

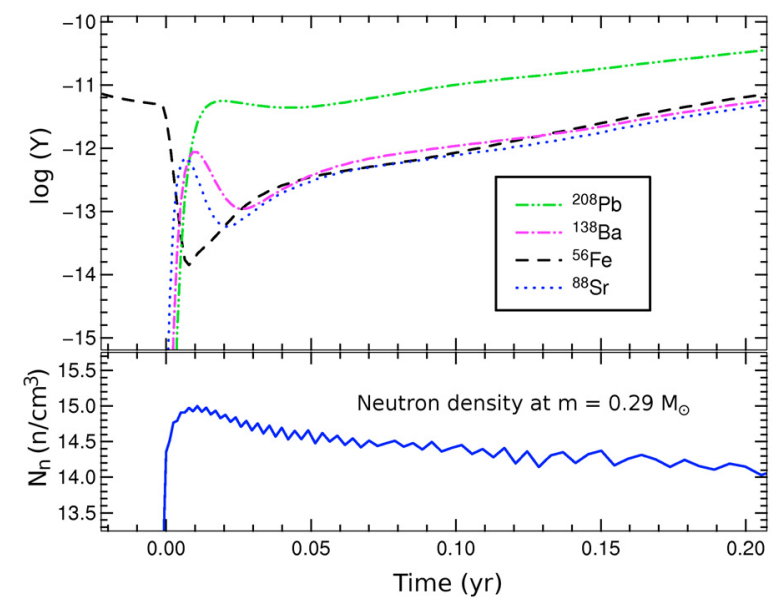

Fig. 3. Evolution of the neutron density (lower panel, in logarithm) and of the abundances of selected heavy element species (upper panel, in logarithm) as a function of time during the main phase of the neutron superburst $(t=0$ corresponds to the same time as in Figs. 1 and 2 and to the onset of the neutron flux). Abundances are sampled at $0.29 M_{\odot}$, the location of the maximum neutron density. All abundances, except those of protons and neutrons, are homogeneous within the $\mathrm{HeCZ}$, and therefore their values in the plot are representative of the abundances in the whole region.

the location of the peak neutron density, at $0.29 M_{\odot}$ in mass, and for neutron densities $>10^{12} \mathrm{n} \mathrm{cm}^{3}$, the temperature decreases in time from 212 to $160 \mathrm{MK}$ and the gas density from 15200 to $2900 \mathrm{~g} \mathrm{~cm}^{-3}$. Free neutrons are available for neutron captures that result in efficient production of the elements heavier than iron and up to lead (Fig. 3). The large total neutron flux in combination with the long time scale for the release of the neutrons result in elemental abundances quite typical of the $s$ process at low metallicity. We find production of nuclei at the three $s$-process peaks corresponding to the magic number of neutrons 50,82 , and 126 , as represented by the isotopes of ${ }^{88} \mathrm{Sr},{ }^{138} \mathrm{Ba}$, and ${ }^{208} \mathrm{~Pb}$ in Fig. 3.

At the start of the neutron burst the abundance of ${ }^{56} \mathrm{Fe}$ is depleted as the neutron capture chain converts the iron into ${ }^{88} \mathrm{Sr}$. After the neutron exposure reaches the value of $\simeq 10 \mathrm{mbarn}^{-1}$, the abundance of ${ }^{88} \mathrm{Sr}$ starts to decrease and that of ${ }^{138} \mathrm{Ba}$ to increase. This is caused by neutrons overcoming the first bottleneck at the magic neutron number $N=50$. Slightly later on, as the neutron exposure reaches $\simeq 20 \mathrm{mbarn}^{-1}$, the same applies to the abundance of ${ }^{138} \mathrm{Ba}$ as the second bottleneck at $N=82$ is overcome and ${ }^{208} \mathrm{~Pb}$ starts to be produced. Once the neutron exposure reaches more than $\simeq 100$ mbarn $^{-1}$, the absolute abundances increase until the end of the neutron flux. By the end of the neutron flux the abundance of ${ }^{56} \mathrm{Fe}$ has grown by a factor of
Table 1. Selected elemental $[\mathrm{X} / \mathrm{Fe}]=\log _{10}(\mathrm{X} / \mathrm{Fe})_{\mathrm{star}}-\log _{10}(\mathrm{X} / \mathrm{Fe})_{\odot}$, except for $\mathrm{Li}$ given as $\left.\log \epsilon(\mathrm{Li})=\log \left(\mathrm{N}_{\mathrm{Li}} / \mathrm{N}_{\mathrm{H}}\right)+12\right)$.

\begin{tabular}{cccc}
\hline \hline Element & $\begin{array}{c}\text { Stellar surface } \\
\text { [model] }\end{array}$ & $\begin{array}{c}f_{\text {DIL }} \simeq 3 \times 10^{-4} \\
{[\text { model] }}\end{array}$ & $\begin{array}{c}\text { HE 1327-2326 } \\
\text { [observations] }\end{array}$ \\
\hline $\log \epsilon(\mathrm{Li})$ & 1.63 & 1.87 & $<0.62$ \\
$\mathrm{C}$ & 5.08 & 3.78 & 3.78 \\
$\mathrm{~N}$ & 4.98 & 3.68 & 4.28 \\
$\mathrm{O}$ & 5.18 & 3.88 & 3.42 \\
$\mathrm{~F}$ & 4.19 & 2.90 & \\
$\mathrm{Na}$ & 2.77 & 1.47 & 2.73 \\
$\mathrm{Mg}$ & 2.17 & 0.87 & 1.97 \\
$\mathrm{Al}$ & 1.48 & 0.18 & 1.46 \\
$\mathrm{Ca}$ & 1.31 & $0.36(0.03)$ & $0.44-0.91$ \\
$\mathrm{Ti}$ & 2.83 & $1.59(0.57)$ & 0.91 \\
$\mathrm{Ni}$ & 1.04 & $-0.04(0.01)$ & 0.18 \\
$\mathrm{Rb}$ & 4.29 & $3.05(1.88)$ & \\
$\mathrm{Sr}$ & 4.19 & $2.94(1.79)$ & 1.17 \\
$\mathrm{Y}$ & 4.32 & $3.07(1.91)$ & \\
$\mathrm{Zr}$ & 4.48 & $3.23(2.06)$ & \\
$\mathrm{Ba}$ & 5.07 & $3.82(2.65)$ & $<1.40$ \\
$\mathrm{La}$ & 5.11 & $3.86(2.69)$ & \\
$\mathrm{Ce}$ & 5.26 & $4.01(2.84)$ & \\
$\mathrm{Nd}$ & 5.03 & $3.79(2.62)$ & \\
$\mathrm{Eu}$ & 4.26 & $3.01(1.90)$ & $<4.64$ \\
$\mathrm{~Pb}$ & 6.25 & $5.00(3.82)$ & \\
\hline
\end{tabular}

232 because of neutron captures on the lighter elements starting from the abundant ${ }^{12} \mathrm{C}$.

\subsection{Surface composition and comparison to the halo star HE 1327-2326}

Dredge-up of the material previously included in the $\mathrm{HeCZ}$ occurs about $10^{4}$ years later, where we find the maximum postflash penetration of the convective envelope. In Table 1 (Col. 2) we show the resultant surface abundances of the model after this dredge-up event. Owing to numerical problems, our current calculations only reached to a time just after the splitting of the convective zone. Thus, in Table 1 the surface abundances of the elements up to $\mathrm{Al}$ have been taken from Paper $\mathrm{I}^{1}$ because they are affected by further proton captures after the convective zone splits, which were not included in the present calculations. The abundances of the elements heavier than $\mathrm{Al}$ are evaluated by considering that $\simeq 0.1 M_{\odot}$ of the material previously involved in the neutron superburst is dredged-up to the stellar surface and diluted with an envelope mass of $0.44 M_{\odot}$, corresponding to a dilution factor $f_{\text {dredge-up }}=0.23$. The surface abundances are then calculated for each nuclear species as mass fractions: $X_{\text {surface }}=\left[X_{\text {initial }}+X_{\mathrm{HeCZ}} \times f_{\text {dredge-up }}\right] /\left[1+f_{\text {dredge-up }}\right]$, where $X_{\text {initial }}$ is the initial envelope value and $X_{\mathrm{HeCZ}}$ is the value in the $\mathrm{HeCZ}$.

It can be seen in Table 1 that neutron-capture elements appear at the surface. Their abundances are highly enriched, in the case of $\mathrm{Pb}$ reaching up to the level of the absolute solar abundance. The high neutron density of the neutron flux contributes to a distribution that is weighed towards neutron-rich nuclei. For example, ${ }^{87} \mathrm{Rb}$ is produced, resulting in $\mathrm{Rb} / \mathrm{Sr} \simeq$ solar. Fe is also increased by 2.2 dex. As reported in Paper I, the model also predicts a large overproduction of $\mathrm{C}, \mathrm{N}, \mathrm{O}$, and $\mathrm{F}$ and milder enhancements of $\mathrm{Na}$ and $\mathrm{Mg}$.

\footnotetext{
1 This is self-consistent because the nuclear species included in our present extended network have so much smaller abundances than the lighter elements that their effect on the overall neutron flux is insignificant.
} 
In Col. 3 of Table 1 we include the composition resulting from diluting the surface composition with material of initial composition by a factor $f_{\text {DIL }} \simeq 3 \times 10^{-4}$. This specific dilution factor was used to match the $\mathrm{C}$ abundance derived from the most metal-poor star discovered to date, the subgiant star HE 1327-2326, with $[\mathrm{Fe} / \mathrm{H}]=-5.96$ (Col. 4 of Table 1 , with typical error bars \pm 0.25 ; Aoki et al. 2006; Frebel et al. 2008). By applying this dilution factor our model self-consistently reproduces the observed $\mathrm{C}, \mathrm{N}$, and $\mathrm{O}$ abundances within a factor of 4 for $\mathrm{N}$ and 3 for $\mathrm{O}$ (observational error bars are $\sim$ a factor of 2), with $\mathrm{Fe}$ increasing by only $20 \%$. However, it produces 59 times more $\mathrm{Sr}$ and at least 260 times more Ba than observed. We performed a preliminary post-processing calculation using an initial $[\mathrm{Fe} / \mathrm{H}]=-5.76$ (numbers in brackets and italics in Col. 3 of Table 1), more similar to HE 1327-2326. We would not expect this relatively small change in the Fe abundance to have a major impact on the overall stellar structure and the nucleosynthesis of the light elements, but we discovered that it has a strong impact on the nett yields of the neutron-capture process, while the relative abundance distribution is not significantly altered. The results from this calculation show a better match to the observed $\mathrm{Sr}$ abundance, within a factor of 4, while still producing too much Ba by a factor of at least 18 . In this case, Fe is unchanged.

The composition of HE 1327-2326 could have arisen from binary system mass-transfer via wind accretion or Roche-lobe overflow from a star such as that modelled here (which would now be a white dwarf). Subsequent dilution of the accreted material in the envelope of HE 1327-2326 via convection or thermohaline mixing would be expected (Stancliffe \& Glebbeek 2008). Because radial velocity variations have so far not been detected for HE 1327-2326, the Roche-lobe overflow mass transfer scenario is not favoured. Wind accretion in a very wide binary remains a possibility and would be more in line with the large dilution factor that we have estimated. The amount of mass that a binary companion of mass $0.8 M_{\odot}$ would need to accrete from our hypothetical primary star to match the observed $\mathrm{C}$ abundance is $\sim 2.4 \times 10^{-4} M_{\odot}$. Here we have assumed the extreme case where the accreted material mixes throughout the entire star. Using this accretion mass together with an estimated total mass loss from the primary star of $0.4 M_{\odot}$ (leaving a $0.6 M_{\odot}$ WD) the expected period of the system is $769 \mathrm{yr}$, using Eq. (12) of Suda et al. (2004), which includes Bondi-Hoyle accretion. This is consistent with the current non-detection of radial velocity variations.

\section{Discussion}

We compared our theoretical surface abundances with the observed abundances of HE 1327-2326. Owing to the high degree of surface pollution in our model only a small amount of mass should have been accreted by HE 1327-2326 to match its composition, indicating a wide binary configuration. The model self-consistently reproduced $\mathrm{C}, \mathrm{N}, \mathrm{O}$, and $\mathrm{Sr}$ within a factor of 4 (if $[\mathrm{Fe} / \mathrm{H}]=-5.76$ ), but overproduced $\mathrm{Ba}$. The model produces more $\mathrm{Sr}$ than $\mathrm{Ba}$ before 0.008 yr (Fig. 3), when the neutron exposure is less than $\simeq 10$ mbarn $^{-1}$. At the first peak of ${ }^{88} \mathrm{Sr}$ we find $[\mathrm{Ba} / \mathrm{Sr}]<0.19$, which would be consistent with the observed $[\mathrm{Ba} / \mathrm{Sr}]<0.23$. However, the absolute $\mathrm{Sr}$ abundance in the $\mathrm{HeCZ}$ at this point is 120 times smaller than the final abundance dredged up (for the $[\mathrm{Fe} / \mathrm{H}]=-5.76$ model), while a factor of $\simeq 17$ smaller could still provide a match to $\mathrm{Sr}$ in HE 1327-2326 within a factor of 4. To match the composition of HE 1327-2326, in this case also including $\mathrm{Ba}$, we would need $\mathrm{C}$, $\mathrm{N}$, and $\mathrm{O} \simeq 7$ times smaller and $f_{\text {DIL }} \simeq 7$ times larger than reported in Table 1 with an expected binary period of $179 \mathrm{yr}$, assuming the same amount of dredge-up. These possibilities may be within the model uncertainties and need to be carefully investigated because the DCF scenario appears to have the potential to self-consistently reproduce most of the strange overabundances in hyper-metal-poor stars (e.g. Schlattl et al. 2001; Picardi et al. 2004; Suda \& Fujimoto 2010, Paper I; Table 1). The DCF would be best studied in the framework of multidimensional models (Deupree 1996; Dearborn et al. 2006; Mocák et al. 2010; Herwig et al. 2010), and effects such as convective overshoot, extra mixing, and rotationally induced mixing should be also investigated in this context.

Our model underproduces $\mathrm{Na}, \mathrm{Mg}, \mathrm{Al}, \mathrm{Ca}, \mathrm{Ti}$, and $\mathrm{Ni}$. This suggests that these elements came with Fe from an early supernova that polluted the protostellar cloud (Joggerst et al. 2010), even though $\mathrm{Na}$ is also underproduced in these models. The uncertainties of the $\mathrm{Ne}$ and $\mathrm{Na}$ proton-capture reactions must be investigated. Lithium is another mismatch because the DCF lowers the surface Li abundance by only a factor of two, while HE 1327-2326 is heavily depleted in Li. This problem needs to be addressed in terms of mixing processes on the secondary star HE 1327-2326.

The peculiar chemical signatures of the DCF event may be able to shed light on star formation and the initial mass function in the early Universe, since the implication of our scenario is that binary systems of roughly solar-mass stars should have formed from hyper-metal-poor gas. We suggest that our results should be taken into account in the study of the composition of extremely metal-poor stars in our halo and nearby galaxies.

Acknowledgements. We appreciate the help of the anonymous referee for the improvement of our discussion. M.L. is a Monash Research Fellow. S.W.C. acknowledges the support of the Consejo Superior de Investigaciones Científicas (CSIC) via a JAE-DOC postdoctoral fellowship. AK is a Stromlo Fellow. Calculations were made on the Australian National Facility supercomputer, under Projects $y 12$ and $g 61$.

\section{References}

Aoki, W., Frebel, A., Christlieb, N., et al. 2006, ApJ, 639, 897

Campbell, S. W. 2007, Ph.D. Thesis, Monash University

Campbell, S. W., \& Lattanzio, J. C. 2008, A\&A, 490, 769

Cyburt, R. H., Amthor, A. M., Ferguson, R., et al. 2010, ApJS, 189, 240

Dearborn, D. S. P., Lattanzio, J. C., \& Eggleton, P. P. 2006, ApJ, 639, 405

Deupree, R. G. 1996, ApJ, 471, 377

Frebel, A., Collet, R., Eriksson, K., Christlieb, N., \& Aoki, W. 2008, ApJ, 684, 588

Fujimoto, M. Y., Iben Jr., I., \& Hollowell, D. 1990, ApJ, 349, 580

Herwig, F., Pignatari, M., Woodward, P. R., et al. 2010, ApJ, submitted

Hollowell, D., Iben Jr., I., \& Fujimoto, M. Y. 1990, ApJ, 351, 245

Iben, Jr., I. 1976, ApJ, 208, 165

Joggerst, C. C., Almgren, A., Bell, J., et al. 2010, ApJ, 709, 11

Lugaro, M., Campbell, S. W., \& de Mink, S. E. 2009, PASA, 26, 322

Meynet, G., Maeder, A., \& Mowlavi, N. 2004, A\&A, 416, 1023

Mocák, M., Campbell, S. W., Müller, E., \& Kifonidis, K. 2010, A\&A, 520, A114

Picardi, I., Chieffi, A., Limongi, M., et al. 2004, ApJ, 609, 1035

Schlattl, H., Cassisi, S., Salaris, M., \& Weiss, A. 2001, ApJ, 559, 1082

Stancliffe, R. J., \& Glebbeek, E. 2008, MNRAS, 389, 1828

Suda, T., \& Fujimoto, M. Y. 2010, MNRAS, 405, 177

Suda, T., Aikawa, M., Machida, M. N., Fujimoto, M. Y., \& Iben, Jr., I. 2004, ApJ, 611, 476 